\title{
The expected photometrical characteristics of high redshift spiral galaxies
}

\author{
M. Mollá ${ }^{1}$, M. García-Vargas ${ }^{2}$ and M. Martín-Manjón ${ }^{3}$ \\ ${ }^{1}$ Dpto. de Investigación Básica, CIEMAT, Avda. Complutense 22, 28040, Madrid (Spain) \\ email:mercedes.molla@ciemat.es \\ ${ }^{2}$ FRACTAL SLNE, C/ Tulipán 2, p13, 1A , 28231 Las Rozas de Madrid (Spain) \\ email:marisa.garcia@fractal-es.com \\ ${ }^{3}$ Dpto. de Física Teórica, Universidad Autónoma de Madrid. 28049 Cantoblanco (Spain) \\ email:mariluz.martin@uam.es
}

\begin{abstract}
The star formation and the subsequent metallic enrichment of spiral galaxies, occurring at the early phases of their evolution, produce emission lines whose contribution may change the colors of these objects. We have computed this contribution with consistent chemo-spectrophotometrical theoretical models in order to calculate the variations in the colors evolution.
\end{abstract}

Keywords. Galaxies: Evolution, Galaxies: Photometry, Galaxies: Abundances

\section{Evolutionary and Photoionization Synthesis Models}

We present the PopStar evolutionary synthesis model in Mollá et al. (2009)(Paper I). Selected isochrones are a new Padova set, specifically computed for this piece of work with a broad age and metallicity coverage, and a detailed treatment of mass-loss for both, young (O, B, WR) and old ages (post-AGB until planetary nebula). The spectral energy distributions (SEDs) are calculated for each Single Stellar Population (SSP) by including the nebular contribution. Colors are calculated for these SEDs for different photometric systems. They result redder for the youngest stellar populations, mainly for low Zmet, than those obtained by Bruzual \& Charlot (2003) but they are similar to those obtained by Leitherer et al. (1999) for ages younger than 1 Gyr. The old stellar populations show the same colors than Bruzual \& Charlot (2003). Both results imply that our models are equally well tuned for young as for old stellar populations.

We have then computed photoionization models (CLOUDY) with the previously described SSP-SEDs, obtaining the emission line spectra due to the youngest stellar populations Martín-Manjón et al. (2009)(Paper II). Some intense emission lines fall in the broad band filters and therefore their contribution must be included into the magnitudes. Colors of stellar clusters changes appreciably, such as we may see in Fig. 3 from Paper III]gv09, in particular when the emission lines proceed from very young stellar clusters but also when they are as old as $10 \mathrm{Myr}$. In the color-color diagrams, when a burst of star formation takes places, points go out of the stellar population region, falling in a region impossible to reach in any other way.

\section{Spiral Evolution Models}

The same basic chemical evolution numerical model was applied to a wide grid of theoretical galaxies with different total masses and variable star formation efficiencies in Mollá \& Díaz (2005). This grid reproduces well the observational data for local 

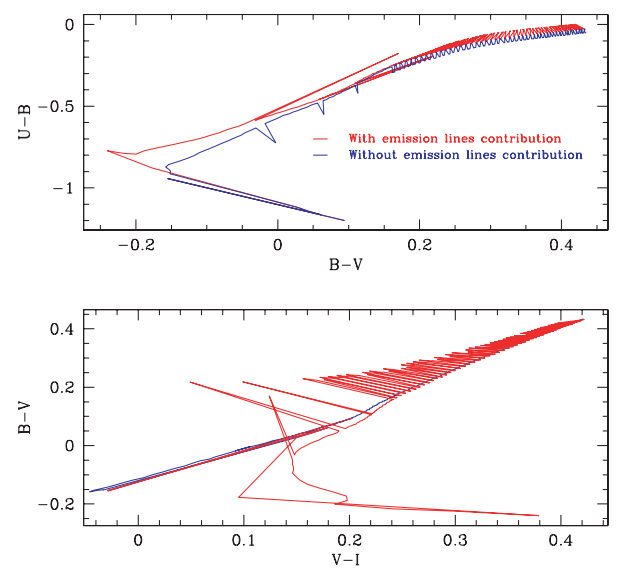

Figure 1. Color-Color diagrams with and without the emission lines contribution

spiral galaxies. By using the resulting star formation, $\Psi(t)$, and metal enrichment, $\mathrm{Z}(\mathrm{t})$, histories, we calculate the SED, $F_{\lambda}(t)$, for each galaxy from the equation:

$$
F_{\lambda}(t)=\int_{0}^{t} S_{\lambda}\left(\tau, Z\left(t^{\prime}\right)\right) \Psi\left(t^{\prime}\right) d t^{\prime},
$$

where $\tau=t-t^{\prime}$ and $S_{\lambda}(\tau, Z)$ is the SED of each stellar generation or SSP of a given age $\tau$ and a metallicity $Z$, taken from Paper I spectra. Once these SEDs calculated, we compute magnitudes and colors in the Johnson and SDSS systems. They reproduce well the data of our local universe, demonstrating that the grid of models is well calibrated.

\section{Results and Conclusions}

We check if the observed colors of galaxies change when emission line intensities are included in the calculations. We compute the observed and rest-frame colors evolution along the redshift for a MWG-type galaxy with continuous star formation. The rest-frame absolute colors U-B and B-V evolution are shown in Fig. 1. The emission lines produced by the last stellar generations change the magnitudes in $0.2 \mathrm{mag}$ in any band.

In summary, the contribution of the emission lines when a burst takes place changes the broad band magnitudes, therefore it is essential to take it into account when starforming galaxies are studied. This is important specially at redshifts in which the star formation history reaches its maximum. Therefore to interpreting high redshift colors by using evolutionary synthesis models without taking into account the star formation history of galaxies and the subsequent emission lines may yield erroneous conclusions.

\section{References}

Bruzual, G. \& Charlot, S. 2003, MNRAS, 344, 1000

García-Vargas, M. L., Mollá, M., \& Martín-Manjón, M. L. 2009, in preparation (Paper III)

Leitherer, C., Schaerer, D., Goldader, J. D., \& Gonzalez Delgado, R. M. et al. 1999, ApJS, 123, 3

Martín-Manjón, M. L., García-Vargas, M. L., Mollá, M., \& Díaz, A. I. 2009, MNRAS, submitted (Paper II)

Mollá, M. \& Díaz, A. I. 2005, A\&A, 359, 18

Mollá, M., García-Vargas, M. L., \& Bressan, A. 2009, MNRAS, 398, 451 (Paper I) 